Running Title: Negative Independent/Dependent Events Scale

\title{
Development and preliminary validation of a self-report measure of negative independent and dependent events in Japanese university students
}

\author{
Akira Hasegawa $^{1 *}$, Shin-ichi Oura ${ }^{1}$, Tetsuya Yamamoto $^{2}$, \\ Yoshihiko Kunisato $^{3}$, and Yoshikazu Fukui ${ }^{4}$ \\ ${ }^{1}$ Faculty of Human Relations, Tokai Gakuin University \\ ${ }^{2}$ Graduate School of Technology, Industrial and Social Sciences, Tokushima University \\ ${ }^{3}$ Department of Psychology, School of Human Sciences, Senshu University \\ ${ }^{4}$ Faculty of Letters, Konan University
}

*Correspondence: Akira Hasegawa

Tokai Gakuin University

5-68 Naka-kirino, Kakamigahara City, Gifu, 504-8511, Japan

e-mail: mail-ad.of.hasse@ tokaigakuin-u.ac.jp

Telephone number: +81-58-389-2200 


\begin{abstract}
We developed a self-report measure for assessing experiences of negative interpersonal dependent events, negative non-interpersonal dependent events, and negative independent events in Japanese university students, which was named the Negative Independent/Dependent Events Scale. We selected items having sufficient content validity in each negative events category. Undergraduate students $(\mathrm{N}=247)$ responded to the Negative Independent/Dependent Events Scale and selfreported measures of depressive symptoms, reassurance-seeking behaviors, inattention, and lack of perseverance. Consistent with our prediction, all the negative events subscales had moderate positive correlations with depressive symptoms. Furthermore, the negative interpersonal dependent events subscale showed a moderate positive correlation with reassurance-seeking behaviors, and the negative non-interpersonal dependent events subscale showed a strong positive correlation with inattention. Moreover, the negative non-interpersonal dependent events subscale was more strongly correlated with inattention than the other two negative events subscales. In contrast, the negative interpersonal dependent events subscale was more strongly correlated with reassurance-seeking behaviors than with the negative independent events subscale but not more strongly than with the negative non-interpersonal dependent events subscale. These findings indicated the acceptable construct validity of the Negative Independent/Dependent Events Scale. However, further research is necessary to establish the discriminant validity of the negative interpersonal dependent events subscale and the negative non-interpersonal dependent events subscale.
\end{abstract}

Keywords: stress generation, negative events, depression, reassurance-seeking, inattention. 
Early studies on stress and depression have mainly focused on the effect of negative life events on depression. Several studies have indicated that the experience of negative events increases the risk of developing depressive symptoms and depressive disorders (for review, Hammen, 2005; Monroe et al., 2014). However, since the seminal work by Hammen (1991), researchers have assumed that depression-prone individuals are not simply passive recipients of negative life events but active agents in the creation of such events. Longitudinal studies based on this assumption named the stress generation hypothesis (Hammen, 1991) have shown that individuals with depressive disorders or subclinical depressive symptoms experience negative events more frequently than their non-depressed counterparts. Furthermore, studies suggest that individuals with depressogenic characteristics such as negative cognitive styles, ruminative response styles, neuroticism, and excessive reassurance-seeking, experience frequent negative events (Liu \& Alloy, 2010, for review). Recent research has also indicated a biological basis for stress generation, including the serotonin transporter-linked polymorphic region genotype (Harkness et al., 2015; Starr et al., 2012) and stress-reactive respiratory sinus arrhythmia (Hamilton \& Alloy, 2017).

Negative life events generated by the self might predict future increases in depressive symptoms (e.g., Belmans et al., 2019; Flynn et al., 2010; Flynn \& Rudolph, 2011; Hankin et al., 2010; Snyder \& Hankin, 2016), and the recurrence of major depression (Bos et al., 2007). Therefore, the stress generation hypothesis can explain person-environment interactions exacerbating depression. Research based on this hypothesis is expected to develop effective interventions for preventing and improving depression by preventing stress generation.

Stress generation studies have often classified negative events into independent events occurring outside the individual's control, such as the death of a relative or friend, and dependent 
events occurring under the influence of the individual's control, such as interpersonal conflicts. Negative events have also been classified as interpersonal events and non-interpersonal events, such as work-related and academic stressors (Hammen \& Shih, 2008; Liu \& Alloy, 2010). It has been reported that individuals vulnerable to depression are likely to experience a higher rate of dependent events, particularly within the interpersonal domain rather than independent events (Liu \& Alloy, 2010, for review). Therefore, it is crucial for stress generation studies to use assessment tools to assess negative independent and negative dependent events separately and identify these events as related to interpersonal or non-interpersonal domains.

Specific studies on stress generation have assessed the experiences of negative events using self-report measures (e.g., Bouchard \& Shih, 2013; Hankin et al., 2010; Liu \& Kleiman, 2012), and other studies have assessed them using interviews (e.g., Flynn \& Rudolph, 2011; Hammen, 1991; Harkness et al., 2015). Assessments using self-report measures compared to interviews have several shortcomings, including the contamination of subjective appraisal of negative events in assessing the actual occurrence of such events and difficulties in collecting detailed contextual information on events for judging whether they are dependent on the individual's control or not, and whether they are interpersonal or not. However, self-report measures of negative events have specific strengths, including the short response time compared to interviews that might take over one hour (Liu, 2013). Therefore, self-report measures are appropriate when researchers use multiple measures for assessing many constructs that take an extended time, and negative events are only one of the constructs of interest. In addition, self-report measures can be used in online surveys, which is not the case with interviews. Considering these benefits, self-report measures of negative events are a useful assessment tool in stress generation research. 
Self-report measures used in previous stress generation studies have specific limitations. Firstly, investigators independently categorized negative events in the scales, which were not explicitly designed to test the stress generation hypothesis, as independent or dependent events and interpersonal or non-interpersonal events. Therefore, different items of the same scale have been used to assess each category of negative events in different studies. For example, Hankin et al. (2010) classified 57 items in the Adolescent Life Events Questionnaire (ALEQ; Hankin \& Abramson, 2002) into 26 interpersonal dependent events, 11 non-interpersonal dependent events, and 13 independent events. Moreover, they excluded 7 items because these items were neither interpersonal nor non-interpersonal. Auerbach et al. (2010), who used the ALEQ composed of the identical 57 items, classified 29 items as interpersonal dependent events. On the other hand, Belmans et al. (2019) used only 18 items as interpersonal dependent events, although it is uncertain whether they used the identical ALEQ items as Hankin et al. (2010) and Auerbach et al. (2010). Using different items to assess each category of negative event experiences has made it difficult to compare findings between studies.

Furthermore, the validity of currently available negative events scales has not been adequately investigated in the stress generation research context. Researchers have merely selected negative interpersonal dependent event items, negative non-interpersonal dependent event items, and negative independent event items by considering their content validity. However, the construct validity of these scales has not been examined to date. Therefore, a need remains to develop a scale with high construct validity that can specifically assess each category of negative events.

To our knowledge, there has been no attempt in Japan to assess negative dependent events and negative independent events separately. For example, Hasegawa et al. (2021) examined whether response inhibition deficits increased rumination through the generation of negative 
interpersonal events in Japanese university students. However, they did not use negative interpersonal dependent event items selected in the stressors scale in their analyses because the interpersonal events subscale they used consisted of only 15 items. Developing a measure for specifically assessing different event categories in the context of stress generation would help identify determinants and consequences of stress generation. Furthermore, it is necessary to develop a scale of negative events suitable for peoples' life stage because negative events that people tend to experience differ depending on their life stage.

Considering the above issues, in this study, we developed a scale to separately assess the experiences of negative interpersonal dependent events, negative non-interpersonal events, and negative independent events in Japanese university students. A scale for assessing negative events experienced by university students was developed because university students are highly targeted populations in stress generation research (e.g., Bouchard \& Shih, 2013; Flynn et al., 2010; Joiner et al., 2005; Liu \& Kleiman, 2012). This scale was named the Negative Independent/Dependent Events Scale. Items with high content validity for the three subscales were selected among an item pool of existing scales. We also examined the correlations between each negative events subscale and variables assumed to be related to the respective event categories to test the scale's construct validity.

We tested the following hypotheses to examine the construct validity of the Negative Independent/Dependent Events Scale. Negative interpersonal dependent events, negative noninterpersonal events, and negative independent events are stressful. Therefore, we hypothesized a moderate or a strong positive correlation between all the event categories and depressive symptoms (Hypothesis 1). 
Moreover, a previous study has indicated that reassurance-seeking behaviors were specifically associated with negative interpersonal dependent events (Stroud et al., 2018). Therefore, we hypothesized a moderate or strong positive correlation between negative interpersonal dependent events and reassurance-seeking behaviors, which is stronger than the correlation between reassurance-seeking behaviors and negative non-interpersonal dependent events or negative independent events (Hypothesis 2).

Furthermore, university students with increased inattention symptoms caused by ADHD are likely to have academic problems (Norwalk et al., 2009; Pope, 2010; Schwanz et al., 2007). Academic problems are a large part of negative non-interpersonal dependent events experienced by university students (see items of negative non-interpersonal dependent events in the Results section). Therefore, we hypothesized a moderate or strong positive correlation between negative non-interpersonal dependent events and inattention and that the correlation between negative noninterpersonal dependent events and inattention is stronger than the correlations between other types of negative events and inattention (Hypothesis 3).

Similarly, university students with high lack of perseverance scores, which is a subdimension of self-reported impulsivity reflecting not following through with a task (Lynam et al., 2006), might have difficulties in completing academic assignments. Therefore, it was hypothesized that a moderate or a strong positive correlation is observed between negative noninterpersonal dependent events and the lack of perseverance, and that the correlation between negative non-interpersonal dependent events and the lack of perseverance is stronger than those between other negative events and the lack of perseverance (Hypothesis 4).

Finally, we attempted to reduce the items of the Negative Independent/Dependent Events Scale because a short scale would allow assessing participants' experiences of negative events in 
a short time. We selected items with a relatively normal distribution of scores and high construct validity for the short scale. This study was conducted under the unusual situation of the global COVID-19 pandemic. Therefore, we could only present preliminary items for the short scale. These items remain to be further validated in future studies.

\section{Method}

\section{Participants}

Japanese undergraduate students at Tokai Gakuin University and Tokushima Universities in Japan $(N=270)$ participated in this study. Participants were recruited in their psychology classes. All participants who agreed to participate in this study completed the questionnaires in their classes. Participants with missing values, or inappropriate responses, including identical responses to all the items in each questionnaire, were excluded from the analysis. The final sample comprised 247 students (153 men, 93 women, and 1 unreported gender; mean age $=19.18$ years, $S D=3.08$, agerange $18-48$ years).

\section{Measures}

Negative Independent/Dependent Events Scale. This self-report measure was originally developed for this study. An Associate Professor in Japan, specializing in psychology (AH) developed an initial pool of 286 items of negative events by reviewing the literature (Hashimoto, 1997, 2005; Hisata \& Niwa, 1987; Kanner, Coyne, Schaefer, \& Lazarus, 1981; Kikushima, 1999, 2002; Kohn, Lafreniere, \& Gurevich, 1990; Miura \& Kawaoka, 2008; Nishino, Kobayashi, \& Kitagawa, 2009; Okayasu, Shimada, Niwa, Mori, \& Yatomi, 1992; Sakamoto \& Kambara, 1998; Shimono \& Hasegawa, 2018; Takahashi, 2013; Takahira, 1998; Toyama \& Sakurai, 1999). AH and a Full-time Lecturer specializing in psychology (SO) selected the items suitable for assessing negative interpersonal dependent events, negative non-interpersonal dependent events, and 
negative independent events from these 286 items. As a result, we selected 80 items; 29 negative interpersonal dependent event items, 22 negative non-interpersonal dependent event items, and 29 negative independent events.

Then, three different associate professors, a postdoctoral fellow, two graduate students studying for a Master's degree, all of them specializing in psychology, and two clinical psychologists with Master's degrees, were asked to rate the 80 items described above. Each event was rated on the following dimensions: (1) the relationship to interpersonal events rated on a 5point scale ranging from 1 (not an interpersonal event at all) to 5 (highly interpersonal event), and (2) the relationship to students' behaviors, personality, and attitudes (i.e., a dependence rating) rated on a 5-point scale ranging from 1 (not related at all or completely independent) to 5 (highly related). We calculated the mean scores of the two ratings for each item. As a result, 25 items among the negative interpersonal dependent event items with a mean score of 3.5 or above for the interpersonal events rating and a mean score of 3 or more for the dependence rating were selected as the final negative interpersonal dependent event items. Similarly, 14 items among the negative non-interpersonal dependent event items with a mean score of 2.5 or less for interpersonal events rating and 3 or more for dependence ratings were selected as the final items. Furthermore, 20 items among negative independent events items with a mean score of 2 or less for dependence rating were selected as the final items.

Participants were requested to respond to the extent to which they had experienced each event in the last 8 weeks using a rating scale ranging from 1 (never) to 4 (often). We set an interval of 8 weeks because we planned to conduct an 8-week longitudinal study subsequently.

Beck Depression Inventory-Second Edition (Beck et al., 1996). This scale is a wellvalidated questionnaire assessing the severity of depressive symptoms experienced in the past two 
weeks. Participants respond to 21 items using a scale ranging from 0-3, with higher scores indicating severe depression. We used the Japanese translation by Kojima and Furukawa (2003) in this study. This study showed the excellent internal consistency of the scale $(\alpha=.93)$.

Revised Japanese Version of the Reassurance-Seeking Scale (Katsuya, 2004). This scale is design to assess behaviors and the desire to seek reassurance. The good reliability and construct validity of the scale has been reported (Katsuya, 2004). The reassurance-seeking behaviors subscale, which is composed of 6 items, was used in this study. Each item was rated on a 7-point rating scale anchored between 1 (not at all true of me) and 7 (entirely true of me). The sufficient internal consistency of the scale was demonstrated in this study $(\alpha=.82)$.

Adult ADHD Self-Report Scale (Kessler et al., 2005). This is a self-report measure designed to assess adults' ADHD symptoms. The Japanese translation of the scale by Takeda et al. (2017) was used in this study. The Adult ADHD Self-Report Scale has demonstrated good reliability and construct validity (Adler et al., 2006; Kessler et al., 2007; Takeda et al., 2017). The scale is composed of two subscales, and we only used the inattention subscale. The scale inquires the frequency of symptoms over the past six months using 9 items, which are rated on a 5-point rating scale anchored between 0 (never) and 4 (very often). The satisfactory internal consistency of the inattention subscale was demonstrated in this study $(\alpha=.84)$.

UPPS-P Impulsive Behavior Scale (Lynam et al., 2006). This is a measure assessing five impulsivity-related traits. We used the Japanese translation by Hasegawa et al. (2018). The UPPSP Impulsive Behavior Scale has reported good reliability and construct validity (Cyders, 2013; Cyders et al., 2007; Hasegawa et al., 2018; Whiteside et al., 2005). Only the lack of perseverance subscale was used in this study. The 10 items of this subscale were rated on a 4-point rating scale 
anchored between 1 (disagree strongly) and 4 (agree strongly). This study demonstrated the acceptable internal consistency of this subscale $(\alpha=.76)$.

\section{Procedure}

This study was conducted from July to August 2020. Since February 2020, COVID-19 had spread in Japan, and all classes in Tokai Gakuin University and Tokushima University had been conducted online until May 2020. When this study was conducted, students in both universities took classes on campus for at least six weeks.

Students were explained about the study before their participation. Only the students that agreed to take part in the study responded to the questionnaires. The Ethics Committee of Tokai Gakuin University approved this study.

\section{Statistical Analysis}

Analyses were conducted on raw data and allowed for missing data. Descriptive statistics were conducted using SPSS ver. 23 (IBM Corporation), and the Z-test was conducted using R ver. 4.0.0. Other analyses were conducted using Mplus 8.3 (Muthén \& Muthén, 1998-2017). Zeroorder Pearson's correlations were computed between each measure, and using the Z-test to compare two dependent correlations was conducted using the procedure described by Hittner et al. (2003). Missing data were handled with multiple imputations using Bayesian analysis when conducting correlation analyses. We inputted all the items of the scales described in the Measures section, and 20 data sets were generated and used for the analyses. We refer to the magnitude of the correlations, using Cohen's (1988) classification $(r<.10$ : negligible association; $.10 \leq r<.30$ : weak association; $.30 \leq r<.50$ : moderate association; $r \geq .50$ : strong association). When comparing two dependent correlations, we applied the Bonferroni correction to divide the significance level of the $p$-value by three (the number of all pairs of correlations; $p=.05 / 3=.017$ ). 
Negative Independent/Dependent Events Scale 12

\section{Results}

\section{Descriptive statistics}

Descriptive statistics of each measure are shown in Table 1. All the study variables' scores, including the three subscales of negative events, were normally distributed. Alpha coefficients were .91 for negative interpersonal dependent events, .80 for negative non-interpersonal dependent events, and .81 for negative independent events.

\section{Construct Validity of the Negative Independent/Dependent Events Scale}

Correlations between each measure are shown in Table 2. Results indicated strong, significant positive correlations among negative interpersonal dependent events, negative noninterpersonal dependent events, and negative independent events. All the negative events subscales indicated significant and moderate positive correlations with depression and reassurance-seeking behaviors. Negative non-interpersonal dependent events showed a strong positive correlation with inattention, whereas negative interpersonal dependent events and negative independents events displayed moderate correlations with inattention. Furthermore, negative interpersonal dependent events and negative non-interpersonal dependent events indicated a significant but weak positive correlation with the lack of perseverance, whereas negative independent events showed a nonsignificant correlation with the lack of perseverance.

The Z-test showed that the correlations of both negative dependent events with depression were stronger than that of negative independent events with depression $(q s>0.14, z s>2.73, p s$ $<.007)$, but the differences in the correlations with depression was not significant between negative interpersonal dependent events and negative non-interpersonal events $(q=0.02, z=0.34$, $p=.732$ ). The correlation of negative interpersonal dependent events with reassurance-seeking behaviors was stronger than that of negative independent events $(q=0.15, z=2.67, p=.008)$, but 
the differences in the correlations with reassurance-seeking behaviors was non-significant between other pairs of negative events ( $q s<0.11, z s<1.87, p s>.061)$. The correlation of negative noninterpersonal dependent events with inattention was stronger than those of the other two negative events $(q s>0.22$, $z s>3.84, p s<.001)$, whereas the difference in the correlations with inattention was not significant between negative interpersonal dependent events and negative independent events $(q=0.09, z=1.59, p=.112)$. The differences in the correlation with lack of perseverance were not significant between any pair of negative events $(q<0.13, z<2.33, p>.020)$.

\section{Selection of appropriate items for the short scale}

The Negative Independent/Dependent Events Scale is composed of a large number of items (i.e., 59 items), and therefore, it is desirable to develop a short version of the scale. We considered it inappropriate to identify the items for the short scale using only the current study's data because this study was conducted under the unusual circumstances of the COVID-19 pandemic and used only three validity scales. Therefore, we have only presented tentative items for the short scale in this study.

We conducted supplementary analyses to identify the appropriate items for each subscale of a short scale. Each item was evaluated on their score distribution because items with skewed distributions are inappropriate for assessing individual differences in experiencing different event categories. We considered items with skewness of 2.00 or over or curtsies of 4.00 or over as inappropriate. In addition, each item was also evaluated for their correlations with reassuranceseeking behaviors and inattention. We considered that items having a correlation of less than .20 with reassurance-seeking behaviors were inappropriate for assessing negative interpersonal dependent events. Moreover, items with a correlation of less than .20 with inattention were considered inappropriate for assessing negative non-interpersonal dependent events. Furthermore, 
items having correlations of .20 or over with reassurance-seeking behaviors and inattention were considered inappropriate for assessing negative independent events.

Among the items, 15 items were highly skewed (6 negative interpersonal dependent events items, 2 negative non-interpersonal dependent events items, and 7 negative independent events items; See Table 3-5). Among the remaining items, 4 negative interpersonal dependent events items had correlations of less than .20 with reassurance-seeking behaviors, and 4 negative independent events items had correlations of .20 or more with reassurance-seeking behaviors and inattention (See Table 3-5).

We summed the scores of the items of each subscale after excluding 23 items described above. Mean scores (SDs) of each subscale were 25.81 (8.19) for negative interpersonal dependent events, 22.72 (5.61) for negative non-interpersonal dependent events, and 15.21 (4.17) for negative independent events. Skewness and Kurtosis were 0.53 and -0.56 , respectively for negative interpersonal dependent events, 0.64 and 0.49 , respectively for negative non-interpersonal dependent events, and 0.54 and -0.15 , respectively for negative independent events. Cronbach's alphas were $.90, .79$, and .65 , respectively for negative interpersonal dependent events, negative non-interpersonal dependent events, and negative independent events.

We also calculated the correlation coefficients among the three subscales and correlation coefficients of each subscale with reassurance-seeking behaviors, inattention, and lack of perseverance. As shown in Table 6, the correlation among each short scale's subscale and the correlations of negative independent events subscale with the validity scales were slightly weaker than the original Negative Independent/Dependent Events Scale. The Z-test indicated that correlations of both negative dependent events and depression were stronger than that of negative independent events $(q s>0.20, z s>3.13, p s<.002)$, while differences in the correlations of 
negative interpersonal dependent events and negative non-interpersonal events with depression was not significant $(q=0.08, z=1.44, p=.149)$. The correlations of both negative dependent events with reassurance-seeking behaviors were stronger than that of negative independent events $(q s>0.17, z s>2.58, p s<.010)$, although the differences in correlations of negative interpersonal dependent events and negative non-interpersonal events with reassurance-seeking behaviors were not significant $(q=0.10, z=1.65, p=.099)$. Moreover, the correlations of negative noninterpersonal dependent events with inattention were stronger than those of the other two types of negative events $(q s>0.18, z s>3.01, p s<.003)$, and the correlation of negative interpersonal dependent events with inattention was stronger than that of negative independent events $(q=0.23$, $z=3.40, p<.001)$. Finally, the differences in correlations with the lack of perseverance among the three subscales were not significant $(q s<0.14, z s<2.14, p s>.033)$.

\section{Discussion}

This study examined the construct validity of the Negative Independent/Dependent Events Scale that specifically assesses the experiences of negative interpersonal dependent events, negative non-interpersonal events, and negative independent events in Japanese university students. Consistent with Hypothesis 1, all the subscales of negative events showed moderate positive correlations with depressive symptoms. This finding confirmed the assumption that events assessed by all the negative events subscales are stressful.

Results also showed that both types of negative dependent events were more strongly correlated with depressive symptoms than negative independent events, although this was not the primary investigation of this study. These results might have been obtained because depressive symptoms generate negative dependent events, but not independent events, as shown in many previous studies (Liu \& Alloy, 2010 for review). However, this cross-sectional study could not 
rule out the possibility that negative dependent events are more detrimental to depression than negative independent events. A longitudinal study undertaken in the future could suggest reasons for the stronger correlations of negative dependent events with depressive symptoms than the correlation of negative independent events. Such a study could clarify the mechanisms of personenvironment interactions that intensify depressive symptoms.

The present findings are partially consistent with the other hypotheses of this study. Negative interpersonal dependent events had a moderate positive correlation with reassuranceseeking behaviors. However, the magnitude of the correlations with reassurance-seeking behaviors differed significantly only between negative interpersonal dependent events and negative independent events, whereas the magnitude of the correlations between negative interpersonal dependent events and negative non-interpersonal dependent events did not differ significantly. Therefore, Hypothesis 2 was only partially supported.

On the other hand, there was a strong positive correlation of negative non-interpersonal dependent events with inattention, and the correlation of negative non-interpersonal dependent events with inattention was significantly stronger than the correlations of negative interpersonal dependent events and negative independent events with inattention. These results supported Hypothesis 3. However, negative non-interpersonal events showed only a weak correlation with lack of perseverance, which was inconsistent with Hypothesis 4 that predicted a moderate or strong correlation, although the results supported the direction of the hypnotized correlation.

The weak correlation between negative non-interpersonal events and the lack of perseverance might have been caused by the item composition of the lack of perseverance subscale. Eight of ten items in this subscale are reversed items. Analyzing the content of the reversed items suggested that the high variance of their scores might reflect constructs other than the lack of 
perseverance. For example, one reversed item, "unfinished tasks really bother me" might assess not only the lack of perseverance but also worry about unfinished tasks. Therefore, the reversed items might not be appropriate for assessing the lack of perseverance. In fact, the correlations of each reversed item score for the lack of perseverance and negative non-interpersonal events ranged from -.28 to .24 , with a mean score of .06 , although correlations of the other two items ranged from .31 to .47 with a mean score of .39 .

The findings in this study supported Hypotheses 1 and 3 and partially supported Hypothesis 2. In addition, experts specializing in clinical psychology confirmed that all items of the Negative Independent/Dependent Events Scale were appropriate for assessing each category of events. The findings of this study and the judgment of experts indicated the acceptable construct validity of the scale.

However, the magnitude of positive correlation with reassurance-seeking behaviors did not significantly differ between negative interpersonal dependent events and negative noninterpersonal dependent events. This result suggested that the scale's ability to discriminate between these two event categories might be insufficient, although the magnitude of the correlations with inattention differed significantly between negative interpersonal dependent events and negative non-interpersonal dependent events. As described above, self-report measures have difficulties in collecting detailed contextual information about each negative event, including information on whether these events were interpersonal or not (Liu, 2013). This methodological limitation could lead to reduced discrimination between negative interpersonal dependent events and negative non-interpersonal dependent events. 
A self-report measure might discriminate between each subscale if the measure were composed of more appropriate items. Therefore, it is desirable to select highly appropriate items based on their correlations with several validity scales as described below.

This study selected preliminary items for the short scale based on the distribution of each item score and the correlations with reassurance-seeking behaviors and inattention, which reduced the scale's items from 59 to 36 . The correlations among all subscales in the short scale ( $r s$ ranged from .52 to .66) were smaller than among all the subscales of the longer scale ( $r$ s ranged from .68 to .69). In addition, as we intended, the differences between the correlation of negative interpersonal dependent events with reassurance-seeking behaviors and the correlations of negative non-interpersonal events and negative independent events with reassurance-seeking behaviors were slightly larger in the short scale $(q s=.10$ and .27 , respectively) than in the long one $(q s=.04$ and .15 , respectively). The difference between the correlation of negative noninterpersonal dependent events with inattention and the correlation of negative independent events with inattention was also more extensive in the short scale $(q=.41)$ than in the long scale $(q=.31)$, although the differences between the correlation of negative non-interpersonal dependent events with inattention and the correlation of negative interpersonal dependent events with inattention were slightly smaller in the short scale $(q=.18)$ than in the longer one $(q=.22)$.

These results suggest that the discrimination between the subscales was clearer in the short scale than in the long scale, and the short scale has higher validity than the longer one, although the latter findings might be a methodological artifact. This study was disadvantaged by using only two scales to examine the appropriate items in each category of negative events. In addition, this study was conducted under the unusual situations of the COVID-19 pandemic in Japan. Therefore, the item composition of the short scale was only preliminary. Future studies after the pandemic 
are expected to use more scales examining the construct validity of the Negative Independent/Dependent Events Scale and more appropriate negative events items.

The scale developed in this study was designed to assess the experiences of negative events in Japanese university students. It is unclear whether this scale can be applied for assessing negative events experienced by university students in other countries because the university systems vary from country to country. We hope that researchers in countries other than Japan will investigate whether the Negative Independent/Dependent Events Scale could be applied to university students in their country.

This study developed a scale for separately assessing negative interpersonal dependent events, negative non-interpersonal dependent events, and negative independent events in Japanese university students that were composed of items with sufficient content validity. In addition, this study demonstrated the acceptable construct validity of the new scale. However, the discrimination between negative interpersonal dependent events and negative non-interpersonal was relatively ambiguous. We also selected preliminary items for the short scale, which might be more appropriate for assessing each category of negative events.

To date, little progress has been made in Japanese research on stress generation because of the lack of suitable scales for specifically examining the causes and consequences of stress generation. We are currently making progress with a research project on stress generation in Japanese university students by using the newly developed Negative Independent/Dependent Events Scale. We expect that evidence accumulated through studies using this scale would indicate the mechanism of person-environment interactions that intensify depression. 
Acknowledgment: The authors thank Ayaka Hori, Eriko Kameyama, Haruki Nishimura, Tetsuya Fujimoto, Yuuki Shimono, and Yuko Matsuda for their help on rating the appropriateness of each negative event item, and Naho Suzuki for her help on translating each negative event item into English.

Funding: This study was supported by grants from the Japan Society for the Promotion of Science (18K13333).

Availability of data and material: This study's dataset can be found at the Open Science Framework [https://osf.io/6t4u8/].

\section{Declarations}

Conflicts of Interest: The authors declared that they had no conflicts of interest with respect to their authorship or the publication of this article.

Ethics approval: All procedures performed in studies involving human participants were in accordance with the ethical standards of the institutional and/or national research committee and with the 1964 Helsinki declaration and its later amendments or comparable ethical standards.

Consent to participate: Informed consent was obtained from all individual participants included in the study. Conflicts of Interest: The authors declared that they had no conflicts of interest with respect to their authorship or the publication of this article. 


\section{References}

Adler, L. A., Spencer, T., Faraone, S. V., Kessler, R. C., Howes, M. J., Biederman, J., \& Secnik, K. (2006). Validity of pilot Adult ADHD Self-Report Scale (ASRS) to rate adult ADHD symptoms. Annals of Clinical Psychiatry, 18(3), 145148. https://doi.org/10.1080/10401230600801077

Auerbach, R. P., Eberhart, N. K., \& Abela, J. R. Z. (2010). Cognitive vulnerability to depression in Canadian and Chinese adolescents. Journal of Abnormal Child Psychology, 38(1), 5768. https://doi.org/10.1007/s10802-009-9344-y

Beck, A. T., Steer, R. A., \& Brown, G. K. (1996). Manual for the Beck Depression Inventory-II. Psychological Corporation.

Belmans, E., Bastin, M., Raes, F., \& Bijttebier, P. (2019). Temporal associations between social anxiety and depressive symptoms and the role of interpersonal stress in adolescents. Depression and Anxiety, 36(10), 960-967. https://doi.org/10.1002/da.22939

Bouchard, L. C., \& Shih, J. H. (2013). Gender differences in stress generation: Examination of interpersonal predictors. Journal of Social and Clinical Psychology, 32(4), 424445. https://doi.org/10.1521/jscp.2013.32.4.424

Bos, E. H., Bouhuys, A. L., Geerts, E., van Os, T. W. D. P., \& Ormel, J. (2007). Stressful life events as a link between problems in nonverbal communication and recurrence of depression. Journal of Affective Disorders, 97(1-3), 161169. https://doi.org/10.1016/j.jad.2006.06.011

Cohen, J. (1988). Statistical power analysis for the behavioral sciences (2nd ed.). Lawrence Earlbaum Associates. 
Cyders, M. A. (2013). Impulsivity and the sexes: Measurement and structural invariance of the UPPS-P Impulsive Behavior Scale. Assessment, 20(1), 8697. https://doi.org/10.1177/1073191111428762

Cyders, M. A., Smith, G. T., Spillane, N. S., Fischer, S., Annus, A. M., \& Peterson, C. (2007). Integration of impulsivity and positive mood to predict risky behavior: Development and validation of a measure of positive urgency. Psychological Assessment, 19(1), 107118. https://doi.org/10.1037/1040-3590.19.1.107

Flynn, M., Kecmanovic, J., \& Alloy, L. B. (2010). An examination of integrated cognitiveinterpersonal vulnerability to depression: The role of rumination, perceived social support, and interpersonal stress generation. Cognitive Therapy and Research, 34(5), 456-466. https://doi.org/10.1007/s10608-010-9300-8

Flynn, M., \& Rudolph, K. D. (2011). Stress generation and adolescent depression: Contribution of interpersonal stress responses. Journal of Abnormal Child Psychology, 39(8), 11871198. https://doi.org/10.1007/s10802-011-9527-1

Hamilton, J. L., \& Alloy, L. B., (2017). Physiological markers of interpersonal stress generation in depression. Clinical Psychological Science, 5(6), 911-929. https://doi.org/10.1177/2167702617720211

Hammen, C. (1991). Generation of stress in the course of unipolar depression. Journal of Abnormal Psychology, 100(4), 555-561. https://doi.org/10.1037/0021-843X.100.4.555

Hammen, C. (2005). Stress and depression. Annual Review of Clinical Psychology, 1(1), 293-319. https://doi.org/10.1146/annurev.clinpsy.1.102803.143938

Hammen, C. \& Shih, J. H. (2008). Stress generation and depression. In K. S. Dobson \& D. J. A. Dozois (Eds.), Risk Factor in Depression (pp. 409-428). Academic Press. 
Hankin, B. L., \& Abramson, L. Y. (2002). Measuring cognitive vulnerability to depression in adolescence: Reliability, validity and gender differences. Journal of Clinical Child and Adolescent Psychology, 31(4), 491-504. https://doi.org/10.1207/153744202320802160

Hankin, B. L., Stone, L., \& Wright, P. A. (2010). Corumination, interpersonal stress generation, and internalizing symptoms: Accumulating effects and transactional influences in a multiwave study of adolescents. Development and Psychopathology, 22(1), 217-235. https://doi.org/10.1017/S0954579409990368

Harkness, K. L., Bagby, R. M., Stewart, J. G., Larocque, C. L., Mazurka, R., Strauss, J. S., Ravindran, A., Rector, N. A., Wynne-Edwards, K. E., \& Kennedy, J. L. (2015). Childhood emotional and sexual maltreatment moderate the relation of the serotonin transporter gene to stress generation. Journal of Abnormal Psychology, 124(2), 275287. https://doi.org/10.1037/abn0000034

Hasegawa, A., Kunisato, Y., Morimoto, H., Nishimura, H., \& Matsuda, Y. (2018). Depressive rumination and urgency have mutually enhancing relationships but both predict unique variance in future depression: A longitudinal study. Cogent Psychology, 5(1), Article 1450919. https://doi.org/10.1080/23311908.2018.1450919

Hasegawa, A., Matsumoto, N., Yamashita, Y., Tanaka, K., Kawaguchi, J., \& Yamamoto, T. (2021). Response inhibition deficits are positively associated with trait rumination, but attentional inhibition deficits are not: Aggressive behaviors and interpersonal stressors as mediators. Psychological Research. Advance online publication. https://doi.org/10.1007/s00426-02101537-y 
Hashimoto, T. (1997). Daigakusei ni okeru taijin stress event bunrui no kokoromi [Categorization of interpersonal stress events among undergraduates]. Japanese Journal of Social Psychology, 13(1), 64-75. https://doi.org/10.14966/jssp.KJ00003724759

Hashimoto, T. (2005). Taijin stressor syakudo no kaihatsu [Development of the new scale of interpersonal stressor]. Studies in Humanities (Shizuoka University), 56(1), 45-71.

Hisata, M., \& Niwa, I. (1987). Daigakusei no seikatsu sutoressa sokutei ni kansuru kenkyu: daigakuseiyo seikatsu taiken shakudo no sakusei [A study on measuring life stressors of college students: Development of the college life experiences scale]. Studies in sociology, psychology and education, 27, 45-55.

Hittner, J. B., May, K., \& Silver, N. C. (2003). A Monte Carlo evaluation of tests for comparing dependent correlations. Journal of General Psychology, 130(2), 149-168. https://doi.org/10.1080/00221300309601282

Joiner, T. E., Jr., Wingate, L. R., Gencoz, T., \& Gencoz, F. (2005). Stress generation in depression: Three studies on its resilience, possible mechanism, and symptom specificity. Journal of Social and Clinical Psychology, 24(2), 236253. https://doi.org/10.1521/jscp.24.2.236.62272

Kanner, A. D., Coyne, J. C., Schaefer, C., \& Lazarus, R. S. (1981). Comparison of two modes of stress measurement: Daily hassles and uplifts versus major life events. Journal of Behavioral Medicine, 4(1), 1-39. https://doi.org/10.1007/BF00844845

Katsuya, N. (2004). Kaiteiban juuyou tasya ni taisuru saikakunin keikou syakudo no shinraisei, datousei no kentou [Reliability and validity of Japanese revised version of reassuranceseeking scale]. The Japanese Journal of Psychology, 13(1), 11-20. https://doi.org/10.2132/personality.13.11 
Kendler, K. S., Karkowski, L. M., \& Prescott, C. A. (1999). Causal relationship between stressful life events and the onset of major depression. The American Journal of Psychiatry, 156(6), 837-848. https://doi.org/10.1176/ajp.156.6.837

Kessler, R. C., Adler, L., Ames, M., Demler, O., Faraone, S., Hiripi, E., Howes, M. J., Jin, R., Scnik, K., Spencer, T., Ustun, T. B., \& Walters, E. E. (2005). The World Health Organization adult ADHD self-report scale (ASRS): A short screening scale for use in the general population. Psychological Medicine, 35(2), 245256. https://doi.org/10.1017/S0033291704002892

Kessler, R. C., Adler, L. A., Gruber, M. J., Sarawate, C. A., Spencer, T., \& Van Brunt, D. L. (2007). Validity of the World Health Organization Adult ADHD self-report scale (ASRS) screener in a representative sample of health plan members. International Journal of Methods in Psychiatric Research, 16(2), 52-65. https://doi.org/10.1002/mpr.208

Kikushima, K. (1999). Stressor to social support ga tyuugaku-ji no hutoukou keikou ni oyobosu eikyou [The effects of stressor and social support on non-attendance tendency in junior high school students]. The Japanese Journal of Personality, 7(2), 66-76. https://doi.org/10.2132/jjpjspp.7.2_66

Kikushima, K. (2002). Daigakusei-you stressor syakudo no sakusei: Stress hannou, social support tono kannkei kara [Development of a scale of stressors in university students: Its relationships with stress responses and social support]. Bulletin of Aichi University of Education (Educational Sciences), 51, 79-84.

Kohn, P. M., Lafreniere, K., \& Gurevich, M. (1990). The Inventory of College Student's Recent Life Experiences: A decontaminated hassles scale for a special population. Journal of Behavioral Medicine, 13(6), 619-630. https://doi.org/10.1007/BF00844738 
Kojima, M., \& Furukawa, T. (2003). Manual for the Beck Depression Inventory-II (Japanese translation). Nihon Bunka Kagakusha Co., Ltd.

Liu, R. T. (2013). Stress generation: Future directions and clinical implications. Clinical Psychology Review, 33(3), 406-416. https://doi.org/10.1016/j.cpr.2013.01.005

Liu, R. T., \& Alloy, L. B. (2010). Stress generation in depression: A systematic review of the empirical literature and recommendations for future study. Clinical Psychology Review, 30(5), 582-593. https://doi.org/10.1016/j.cpr.2010.04.010

Liu, R. T., \& Kleiman, E. M. (2012). Impulsivity and the generation of negative life events: The role of negative urgency. Personality and Individual Differences, 53(5), 609-612. https://doi.org/10.1016/j.paid.2012.05.003

Lynam, D. R., Smith, G. T., Whiteside, S. P., \& Cyders, M. A. (2006). The UPPS-P: Assessing five personality pathways to impulsive behavior [Unpublished report]. Purdue University.

Miura, M., \& Kawaoka, F. (2008). Koukousei-you gakkou stressor syakudo (SSS) no sakusei [Development of a school stressor scale for high school students (SSS)]. Japanese Journal of Counseling Science, 41(1), 73-83.

Monroe, S. M., Slavich, G. M., \& Georgiades, K. (2014). The social environment and depression: The roles of life stress. In I. H. Gotlib \& C. L. Hammen (Eds.), Handbook of depression ( $3^{\text {rd }}$ ed.). (p. 296-314). The Guilford Press.

Muthén, L. K. \& Muthén, B. O. (1998-2017). Mplus User’s Guide. Eighth Edition. Muthén \& Muthén.

Nishino, Y., Kobayashi, S., \& Kitagawa, T. (2009). Nichijou stressor ga yokuutsu keikou ni oyobosu eikyou to jiko-kati no yakuwari ni tsuite no juudan-kenkyuu [The effect of daily stressors on the tendency toward depression and the role of self-worth in the psychological 
stress process: A longitudinal study]. The Japanese Journal of Personality, 17(2), 133-143. https://doi.org/10.2132/personality.17.133

Norwalk, K., Norvilitis, J. M., \& MacLean, M. G. (2009). ADHD symptomatology and its relationship to factors associated with college adjustment. Journal of Attention Disorders, 13(3), 251-258. https://doi.org/10.1177/1087054708320441

Okayasu, T., Shimada, H., Niwa, Y., Mori, T., \& Yatomi, N. (1992). Tyuugakusei no gakkou stressor no hyouka to stress hannou tono kannkei [The relationship between evaluation of school stressors and stress responses in junior high school students]. The Japanese Journal of Psychology, 63(5), 310-318. https://doi.org/10.4992/jjpsy.63.310

Pope, D. J. (2010). The impact of inattention, hyperactivity and impulsivity on academic achievement in UK university students. Journal of Further and Higher Education, 34(3), 335-345. https://doi.org/10.1080/0309877X.2010.484053

Sakamoto, S., \& Kambara, M. (1998). A longitudinal study of the relationship between attributional style, life events, and depression in Japanese undergraduates. The Journal of Social Psychology, 138(2), 229-240. https://doi.org/10.1080/00224549809600374

Schwanz, K. A., Palm, L. J., \& Brallier, S. A. (2007). Attention problems and hyperactivity as predictors of college grade point average. Journal of Attention Disorders, 11(3), 368373. https://doi.org/10.1177/1087054707305155

Shimono, Y., \& Hasegawa, A. (2018). Daigakusei no gakugyou ni okeru stress taisyo ga hikikomori-shinwasei ni oyobosu eikyou: Coping houryaku to enjo-yousei koudou wo toriagete [The impact for coping strategies and help-seeking behaviors on the affinity for “hikikomori” among Japanese university students]. Japanese Journal of Cognitive Therapy, $11(1), 80-91$. 
Negative Independent/Dependent Events Scale 28

Snyder, H. R., \& Hankin, B. L. (2016). Spiraling out of control: Stress generation and subsequent rumination mediate the link between poorer cognitive control and internalizing psychopathology. Clinical Psychological Science, $\quad 4(6), \quad$ 10471064. https://doi.org/10.1177/2167702616633157

Starr, L. R., Hammen, C., Brennan, P. A., \& Najman, J. M. (2012). Serotonin transporter gene as a predictor of stress generation in depression. Journal of Abnormal Psychology, 121(4), 810-818. https://doi.org/10.1037/a0027952

Stroud, C. B., Sosoo, E. E., \& Wilson, S. (2018). Rumination, excessive reassurance seeking, and stress generation among early adolescent girls. The Journal of Early Adolescence, 38(2), 139-163. https://doi.org/10.1177/0272431616659559

Takahashi, S. (2013). Taijin stressor syakudo sakusei no kokoromi [Development of the Interpersonal Stressor Scale]. The Japanese Journal of Personality, 21(3), 306-308. https://doi.org/10.2132/personality.21.306

Takahira, M. (1998). Taijin Tassei ryoikibetsu life event syakudo (daigakusei-you) no sakusei to datousei no kentou [Construction of a scale of life events in interpersonal and achievement domains for undergraduate students]. Japanese Journal of Social Psychology, 14(1), 1224. https://doi.org/10.14966/jssp.KJ00004622673

Takeda, T., Tsuji, Y., \& Kurita, H. (2017). Psychometric properties of the Japanese version of the Adult Attention-Deficit Hyperactivity Disorder (ADHD) Self-Report Scale (ASRS-J) and its short scale in accordance with DSM-5 diagnostic criteria. Research in Developmental Disabilities, 63, 59-66. https://doi.org/10.1016/j.ridd.2017.02.011

Toyama, M., \& Sakurai, S. (1999). Daigakusei ni okeru nichijou-teki dekigoto to kenkou joutai no kannkei: Positive na nichijou-teki dekigoto no eikyou wo tyuusin ni [Daily hassles and 
uplifts, and college students' health: Focusing on daily uplifts]. Japanese Journal of Educational Psychology, 47(3), 374-382. https://doi.org/10.5926/jjep1953.47.3_374

Whiteside, S. P., Lynam, D. R., Miller, J. D., \& Reynolds, S. K. (2005). Validation of the UPPS impulsive behaviour scale: A four-factor model of impulsivity. European Journal of Personality, 19(7), 559-574. https://doi.org/10.1002/per.556 


\section{Table 1}

Descriptive statistics of study measures

\begin{tabular}{lcrrrrrr}
\hline & $n$ & $M$ & \multicolumn{1}{c}{$S D$} & \multicolumn{1}{c}{ Range } & Skewness & Kurtosis \\
\hline Negative interpersonal dependent events & 240 & 38.97 & 11.18 & $25-70$ & 0.62 & -0.61 \\
Negative non-interpersonal dependent events & 243 & 25.22 & 6.05 & $14-48$ & 0.67 & 0.46 \\
Negative independent events & 243 & 32.58 & 7.63 & $20-60$ & 0.77 & 0.26 \\
Depression & 240 & 13.65 & 10.63 & $0-52$ & 1.12 & 1.13 \\
Reassurance-seeking behaviors & 244 & 22.58 & 8.08 & $6-40$ & -0.26 & -0.48 \\
Inattention & 242 & 15.68 & 5.77 & $0-34$ & 0.30 & 0.54 \\
Lack of perseverance & 246 & 23.71 & 4.90 & $12-39$ & 0.10 & 0.11 \\
\hline
\end{tabular}




\section{Table 2}

Correlations between variables $(N=247)$

1

2

3

4

1. Negative interpersonal dependent events

2. Negative non-interpersonal dependent events

3. Negative independent events

4. Depression

5. Reassurance-seeking behaviors

.69

$[.63, .76]$

.69

.68

$[.62, .75] \quad[.61, .74]$

.45

$[.35, .55$

.47

.45

$[.37, .57$

$[.35, .55$

.42

$[.32, .52]$

.33

.45

6. Inattention

$[.35, .55]$

.61

$[.53, .69]$

.33

.16

.22 $[.04, .28$

$[.10, .34]$

$[.22, .44]$

$[.22, .44]$

.38

$[.26, .49]$

.10

.43

$[.33, .54]$

.56

5

$[.47, .65]$

.40

7. Lack of perseverance

$[-.02, .23] \quad[.07 ., .31]$

$[.29, .51]$

.10

.38

Note: Absolute correlations that were .16 or greater were significant at $p<.05$. Numbers in parentheses indicate $95 \%$ confidence intervals. 


\section{Table 3}

Negative interpersonal dependent events items (English translations)

\begin{tabular}{|c|c|c|c|c|c|c|}
\hline \multirow[b]{2}{*}{ I had a quarrel with a family member. } & \multicolumn{3}{|c|}{$\begin{array}{l}\text { Reassurance-seeking } \\
\text { behaviors }\end{array}$} & \multicolumn{3}{|c|}{ Inattention } \\
\hline & .29 & $* * *$ & {$[.18, .41]$} & .33 & $* * *$ & {$[.22, .44]$} \\
\hline I disagreed with a close person such as a friend or a lover. & .36 & $* * *$ & {$[.25, .47]$} & .26 & $* * *$ & {$[.14, .38]$} \\
\hline $\begin{array}{l}\text { I was criticized or teased by my friends and colleagues about my } \\
\text { action. }\end{array}$ & .26 & $* * *$ & {$[.14, .38]$} & .11 & & {$[-.01, .24]$} \\
\hline I was criticized by others in my absence. & .25 & $* * *$ & {$[.13, .37]$} & .19 & $* *$ & {$[.06, .31]$} \\
\hline Others were worried about my bad behavior. & .33 & $* * *$ & {$[.22, .44]$} & .33 & $* * *$ & {$[.21, .44]$} \\
\hline Others spoke or acted as if they did not trust me. & .37 & $* * *$ & {$[.26, .48]$} & .29 & $* * *$ & {$[.18, .41]$} \\
\hline I hurt others' feelings. & .33 & $* * *$ & {$[.21, .44]$} & .36 & $* * *$ & {$[.25, .47]$} \\
\hline I was pulled up or warned by my superiors about my bad attitude. & .12 & & {$[-.00, .24]$} & .21 & $* *$ & {$[.09, .33]$} \\
\hline I offended others. & .37 & $* * *$ & {$[.27, .48]$} & .36 & $* * *$ & {$[.25, .47]$} \\
\hline I was ignored by my friends. & .17 & $* *$ & {$[.05, .29]$} & .28 & $* * *$ & {$[.16, .40]$} \\
\hline I had to respond to an acquaintance's action in vain. & .31 & $* * *$ & {$[.19, .42]$} & .33 & $* * *$ & {$[.21, .44]$} \\
\hline I got involved in my friend's troubles or problems. & .19 & $* *$ & {$[.07, .31]$} & .08 & & {$[-.05, .21]$} \\
\hline I was treated coldly when I tried to get the attention of a girl/boy. & .23 & $* * *$ & {$[.11, .35]$} & .10 & & {$[-.02, .23]$} \\
\hline$\underline{\text { I had a quarrel or a bad relationship with my superior. }}$ & .12 & & {$[-.00, .25]$} & .11 & & {$[-.02, .24]$} \\
\hline I couldn't convey what I wanted to say. & .40 & $* * *$ & {$[.29, .51]$} & .42 & $* * *$ & {$[.32, .52]$} \\
\hline$\underline{I \text { was ridiculed by a friend }}$ & .24 & $* * *$ & {$[.13, .36]$} & .26 & $* * *$ & {$[.15, .38]$} \\
\hline My relationship at my part-time job got worse. & .24 & $* * *$ & {$[.12, .36]$} & .15 & $*$ & {$[.03, .28]$} \\
\hline I couldn't get along with my friends. & .25 & $* * *$ & {$[.14, .37]$} & .30 & $* * *$ & {$[.18, .42]$} \\
\hline$\underline{\text { I was attacked when I pointed out his/her bad behavior. }}$ & .17 & $* *$ & {$[.05, .29]$} & .18 & $* *$ & {$[.06, .30]$} \\
\hline I fought or quarreled with a close person, such as a friend or a lover. & .26 & $* * *$ & {$[.15, .38]$} & .20 & $* *$ & {$[.08, .32]$} \\
\hline I broke up with my lover or was turned down by my favorite person. & .06 & & {$[-.06, .19]$} & .07 & & {$[-.05, .20]$} \\
\hline$\underline{\text { I was ignored or refused by my friends. }}$ & .23 & $* * *$ & {$[.11, .35]$} & .25 & $* * *$ & {$[.13, .38]$} \\
\hline I bothered others. & .36 & $* * *$ & {$[.25, .47]$} & .44 & $* * *$ & {$[.34, .54]$} \\
\hline I had a terrible relationship with my friend. & .20 & $* *$ & {$[.08, .32]$} & .24 & $* * *$ & {$[.13, .36]$} \\
\hline I disagreed with my family. & .25 & $* * *$ & {$[.13, .36]$} & .37 & $* * *$ & {$[.26, .48]$} \\
\hline
\end{tabular}

Note: Underlines indicate items with skewness of 2.00 or more, or curtsies of 4.00 or more. Items highlighted in gray indicate correlations of less than .20 with reassurance-seeking behaviors. 


\section{Table 4}

Negative non-interpersonal dependent events items (English translations)

\begin{tabular}{|c|c|c|c|c|c|c|}
\hline \multirow[b]{2}{*}{ It took a long time to finish my assignments and reports. } & \multicolumn{3}{|c|}{$\begin{array}{c}\text { Reassurance-seeking } \\
\text { behaviors }\end{array}$} & \multicolumn{3}{|c|}{ Inattention } \\
\hline & .15 & $*$ & {$[.02, .27]$} & .33 & $* * *$ & {$[.21, .44]$} \\
\hline I could not finish my assignments and reports by the due date. & .09 & & {$[-.04, .21]$} & .27 & $* * *$ & {$[.15, .39]$} \\
\hline I wasn't satisfied with my presentation. & .33 & $* * *$ & {$[.22, .45]$} & .36 & $* * *$ & {$[.25, .47]$} \\
\hline $\begin{array}{l}\text { I was arrested for breaking the law, such as traffic violations or unpaid } \\
\text { rides. }\end{array}$ & .09 & & {$[-.04, .23]$} & .01 & & {$[-.14, .16]$} \\
\hline I left my stuff outside the house. & .27 & $* * *$ & {$[.15, .38]$} & .33 & $* * *$ & {$[.21, .44]$} \\
\hline I broke or lost something important. & .23 & $* * *$ & {$[.12, .35]$} & .31 & $* * *$ & {$[.19, .42]$} \\
\hline I didn't have enough money or was in debt. & .26 & $* * *$ & {$[.15, .38]$} & .31 & $* * *$ & {$[.20, .43]$} \\
\hline I could not improve at my hobbies or lessons as expected. & .21 & $* *$ & {$[.09, .33]$} & .32 & $* * *$ & {$[.20, .43]$} \\
\hline I had bad exam results. & .18 & $* *$ & {$[.06, .30]$} & .24 & $* * *$ & {$[.12, .35]$} \\
\hline I have many tasks left to do, including writing reports. & .24 & $* * *$ & {$[.12, .36]$} & .47 & $* * *$ & {$[.37, .57]$} \\
\hline I was in trouble about finding a job. & .20 & $* *$ & {$[.08, .32]$} & .24 & $* * *$ & {$[.12, .36]$} \\
\hline The professor gave me a low grade for my class presentation. & .25 & $* * *$ & {$[.14, .37]$} & .32 & $* * *$ & {$[.21, .43]$} \\
\hline My report was badly graded. & .22 & $* * *$ & {$[.10, .34]$} & .30 & $* * *$ & {$[.19, .42]$} \\
\hline I couldn't learn, study, or graduate well. & .32 & $* * *$ & {$[.20, .43]$} & .49 & $* * *$ & {$[.40, .59]$} \\
\hline
\end{tabular}

Note: Underlines indicate items with skewness of 2.00 or more or curtsies of 4.00 or more. Items highlighted in gray indicate a correlation of less than .20 with inattention. 


\section{Table 5}

Negative independent events items (English translations)

\begin{tabular}{|c|c|c|c|c|c|c|}
\hline \multirow[b]{2}{*}{ The neighborhood was noisy. } & \multicolumn{3}{|c|}{$\begin{array}{l}\text { Reassurance-seeking } \\
\text { behaviors }\end{array}$} & \multicolumn{3}{|c|}{ Inattention } \\
\hline & .16 & $*$ & {$[.04, .28]$} & .14 & $*$ & {$[.02, .26]$} \\
\hline I got into trouble with my relatives. & .18 & $* *$ & {$[.06, .31]$} & .17 & $* *$ & {$[.05, .30]$} \\
\hline I faced a disasters such as heavy rain or snow. & .15 & $*$ & {$[.03, .27]$} & .07 & & {$[-.05, .20]$} \\
\hline $\begin{array}{l}\text { I had to wait for a long time in government offices, stores, or banks, } \\
\text { etc. }\end{array}$ & .12 & & {$[-.01, .24]$} & .25 & $* * *$ & {$[.13, .37]$} \\
\hline I was treated coldly in government offices, stores, or banks, etc. & .12 & & {$[-.00, .25]$} & .02 & & {$[-.11, .15]$} \\
\hline A family member or a close person was ill, injured, or died. & .13 & $*$ & {$[.01, .26]$} & .11 & & {$[-.02, .23]$} \\
\hline I was the victim of a crime such as theft or being molested. & .07 & & {$[-.06, .19]$} & .02 & & {$[-.10, .15]$} \\
\hline People did something insane to me. & .26 & $* * *$ & {$[.14, .38]$} & .29 & $* * *$ & {$[.18, .41]$} \\
\hline The event that I was looking forward to was canceled or postponed. & .16 & $*$ & {$[.03, .28]$} & .20 & $* *$ & {$[.08, .32]$} \\
\hline I was forced to practice hard in my club or circle activities. & .12 & & {$[-.01, .24]$} & .11 & & {$[-.01, .24]$} \\
\hline $\begin{array}{l}\text { University facilities, including cafeterias and toilets, were } \\
\text { inconvenient. }\end{array}$ & .25 & $* * *$ & {$[.13, .37]$} & .23 & $* * *$ & {$[.12, .35]$} \\
\hline My friend or a lover has moved far away. & .06 & & {$[-.06, .19]$} & -.02 & & {$[-.15, .11]$} \\
\hline A product I purchased was defective. & .16 & $*$ & {$[.03, .28]$} & .19 & $* *$ & {$[.06, .32]$} \\
\hline I had many exams and assignments imposed on me in classes. & .21 & $* *$ & {$[.09, .33]$} & .32 & $* * *$ & {$[.21, .43]$} \\
\hline My family's household budget has shrunk. & .11 & & {$[-.01, .24]$} & .19 & ** & {$[.06, .31]$} \\
\hline I had an unexpected expense. & .24 & $* * *$ & {$[.12, .35]$} & .38 & $* * *$ & {$[.27, .48]$} \\
\hline Classroom discipline including being quiet in class was not observed. & .15 & $*$ & {$[.02, .27]$} & .16 & $*$ & {$[.03, .28]$} \\
\hline I heard of a problem in my family. & .23 & $* * *$ & {$[.11, .35]$} & .33 & $* * *$ & {$[.22, .44]$} \\
\hline $\begin{array}{l}\text { Public transportation such as trains or buses I used were canceled or } \\
\text { delayed. }\end{array}$ & .13 & $*$ & {$[.01, .26]$} & .11 & & {$[-.02, .23]$} \\
\hline There was a traffic jam. & .09 & & {$[-.04, .21]$} & .13 & $*$ & {$[.01, .25]$} \\
\hline
\end{tabular}

Note: Underlines indicate items with skewness of 2.00 or more or curtsies of 4.00 or more. Items highlighted in gray indicate correlations of .20 or more with reassurance-seeking behaviors and inattention. 
Negative Independent/Dependent Events Scale 35

\section{Table 6}

Short Negative Independent/Dependent Events Scale's correlations with other scales $(N=247)$

\begin{tabular}{lccc}
\hline & 1 & 2 & 3 \\
\hline 1. Negative interpersonal & \multicolumn{3}{c}{} \\
dependent events (15 items) & \multicolumn{3}{c}{} \\
2. Negative non-interpersonal & .66 & & \\
dependent events (12 items) & {$[.59, .73]$} & - & \\
3. Negative independent & .53 & .52 & - \\
events (9 items) & {$[.44, .62]$} & {$[.43, .61]$} & \\
& .51 & .45 & .27 \\
4. Depression & {$[.42, .61]$} & {$[.35, .55]$} & {$[.15, .38]$} \\
& .49 & .41 & .26 \\
5. Reassurance-seeking & {$[.39, .58]$} & {$[.31, .51]$} & {$[.14, .38]$} \\
behaviors & .48 & .61 & .29 \\
& {$[.39, .58]$} & {$[.53, .69]$} & {$[.18, .41]$} \\
6. Inattention & .16 & .20 & .07 \\
7. Lack of perseverance & {$[.04, .28]$} & {$[.08, .32]$} & {$[-.06, .19]$} \\
\hline
\end{tabular}

Note: Absolute correlations greater than .16 are significant at $p<.05$.

Numbers in parentheses indicate $95 \%$ confidence intervals. 